\title{
Endoscopic electrocautery incision therapy for benign lower gastrointestinal tract anastomotic strictures
}

\author{
Deepanshu Jain ${ }^{a}$, Naemat Sandhub ${ }^{b}$, Shashideep Singhalc
}

Albert Einstein medical center, Philadelphia, PA; University of Texas Health Science Center at Houston, USA

\begin{abstract}
Benign anastomotic strictures can occur in up to $22 \%$ of patients who undergo colonic or rectal resection. Traditionally, surgery was the preferred method of treatment, but, over time, endoscopic techniques, such as balloon dilation, have become the preferred modality. However, a high stricture recurrence rate of up to $18-20 \%$ and the increased risk of perforation due to uncontrolled stretching are its major drawbacks. Endoscopic electrocautery incision (EECI) allows for controlled mucosal incision in predetermined locations of stricture. In this meta-analysis, we have summarized case reports, case series, retrospective studies and prospective studies describing the different endoscopic EECI techniques used for benign lower gastrointestinal tract anastomotic strictures. Our analysis showed that EECI, either alone or in combination with other modalities (e.g. balloon dilation, steroid injection or argon plasma coagulation) is an effective treatment option for both treatment-naïve and refractory short non-inflammatory strictures. The overall success rate for EECI-based therapy for benign colorectal stricture was $98.4 \%$, with a stricture recurrence rate of $6.0 \%$. No major adverse event (bleeding, infection or perforation) was reported. Only minor adverse events (abdominal pain) were reported in $3.8 \%$ of the population.
\end{abstract}

Keywords Benign stricture, colorectal stricture, endoscopic incision therapy

Ann Gastroenterol 2017; 30 (4): 1-13

\section{Introduction}

Benign anastomotic strictures can occur in up to $22 \%$ of patients who undergo colonic or rectal resection [1]. The incidence of postoperative colorectal strictures has risen over the years, partly as a result of an increase in the use of stapler devices for anastomotic closure [2]. The treatment of anastomotic strictures is imperative, to limit the risk of development of metachronous cancer lesions over time in patients with prior colorectal cancers and to avoid progression towards obstructive symptoms.

Traditionally, surgery was the preferred method of treatment of colorectal strictures. Over time, that has been

${ }^{\mathrm{a} D i v i s i o n}$ of Gastroenterology, Department of Internal Medicine (Deepanshu Jain); ${ }^{\mathrm{b}}$ Department of Internal Medicine (Naemat Sandhu), Albert Einstein Medical Center, Philadelphia, PA; ${ }^{\mathrm{C}}$ Division of Gastroenterology, Hepatology and Nutrition, University of Texas Health Science Center at Houston, Houston, Texas (Shashideep Singhal), USA

Conflict of Interest: None

Correspondence to: Shashideep Singhal, MD, Division of Gastroenterology, Hepatology and Nutrition, University of Texas Health Science Center at Houston, 6431 Fannin, MSB 4.234, Houston, Texas, USA 77030, e-mail: sdsinghal@gmail.com

Received 27 February 2017; accepted 16 May 2017; published online 30 May 2017

DOI: https://doi.org/10.20524/aog.2017.0163 replaced by endoscopic techniques, primarily balloon dilations and dilation with Savary-Gilliard bougies, which have been demonstrated as effective, simple, and safe [3]. However, in some cases the stricture can be inaccessible, or it can fail to improve with multiple sessions of dilatation. This technique also has a high stricture recurrence rate, up to $18-20 \%$, and carries an increased risk of perforation as a result of uncontrolled stretching [3].

Endoscopic electrocautery incision (EECI) techniques have been described in the literature as alternative therapies for anastomotic strictures, or for resistant strictures that have failed treatment with dilatation. Electrocautery incision creates a defined mucosal incision at the most resistant location and leads to dilation of the stricture. These techniques have been reported to have a good success rate and a lower risk of adverse events, including perforation.

In this systematic review, we have summarized case reports, case series, retrospective studies and prospective studies describing the different EECI techniques used in colonic, rectal or colorectal benign anastomotic strictures.

\section{Materials and methods}

An extensive English language literature search was carried out of studies published until July 2016. PubMed and Google Scholar were used to identify the peer-reviewed original and review articles, based on the following key words: benign 
stricture, colorectal stricture, endoscopic incision therapy. Only human study subject articles were selected. The references of pertinent studies were searched manually to identify additional relevant studies. The search yielded case reports [4-6], case series [7-9], and prospective studies [10-13]. The stricture etiology, characteristics and location, device details, procedure details, success rate, complications and limitations were reviewed for each study.

\section{Results}

Ten original articles were considered appropriate to be included in this review article [4-13]. Three studies described the application of EECI: a case report from Germany [4], a case series from Korea [7] and a retrospective case series from Italy [9]. Three studies described an endoscopic electrocautery radial incision and cutting (RIC) technique: one case report from Brazil [5], a retrospective case series from Japan [8] and a prospective study from Italy [10]. A case report from USA described the use of adjunctive corticosteroids with EECI [6]. Two prospective studies described combination therapy with EECI and balloon dilation: one each from Japan [11] and Germany [12]. Another prospective study from Germany [13] described the role of combination therapy with EECI and Argon plasma coagulation (APC). All studies have been summarized in Table 1.

\section{Discussion}

\section{Stricture}

\section{Etiology and location}

Studies included in this review identified patients with post-surgical anastomotic stricture [4-13]. Post-surgical anastomotic stricture can occur as a result of tissue ischemia, inflammation, hemorrhage at the site of the suture line, anastomotic site leakage or recurrence of disease $[17,18]$. Anastomotic leakage has been shown to contribute towards increased inflammation, thereby contributing to an increased risk of fibrosis and stenosis. According to a Cochrane Database systemic review, a side-to-side stapled anastomosis has been recommended for ileocolic anastomosis, especially following a right hemicolectomy for colonic adenocarcinomas, because it results in fewer anastomotic leaks [20]. A recent meta-analysis for Crohn's disease showed a higher rate of anastomotic leakage with end-to-end anastomosis compared to side-to-side [21].

The spectrum of surgical resections included those for malignant primary lesions, such as low anterior resection for rectosigmoid colon cancer and rectal cancer, intersphincteric resection for lower rectal cancer, hemicolectomy or subtotal colectomy [5,7-13]. Patients undergoing proctocolectomy with ileal-pouch anal anastomosis for ulcerative colitis, sigmoid resection for diverticulitis and volvulus, prophylactic proctocolectomy for familial adenomatous polyposis were also included by some studies in this review $[4,6,9,10]$. Stricture formation across all studies included occurred at the site of the surgical anastomosis [4-13].

\section{Criteria, characteristics and timing}

Anastomotic strictures following colonic and/or rectal resections can present with symptoms of abdominal pain, cramping, constipation or symptoms of bowel obstruction. Studies included in this review identified strictures after patients presented with symptoms or as a result of follow-up assessments. Various radiologic and/or endoscopic techniques were utilized to identify the stricture. Strictures of $<10 \mathrm{~mm}$ diameter were commonly seen to have presented with obstructive symptoms. Strictures in the descending colon or rectum may often present clinically once luminal diameter is $20 \mathrm{~mm}$ or less.

The majority of the studies defined the stricture as the inability to pass an endoscope across the anastomosis site [6-13]. The size of the endoscope used varied across the studies, hence creating a wide variability in the stricture definition. Few studies used radiologic techniques, such as barium enema, barium swallow or CT scan, to define the stricture $[7,10,11,13]$. The diagnosis in these studies was followed by endoscopic confirmation of the stricture. Wallstabe et al [4] used digital palpation and pouchoscopy to identify the area of fibrotic tissue, confirmed on biopsy. Truong et al, in a prospective study of 36 patients, defined three grades of stenosis according to the luminal diameter [12]. The severity of stenosis was closely related to the symptomatic presentation. Symptoms of bowel obstruction correlated with a luminal diameter of $<5 \mathrm{~mm}$, defined as grade- 3 stenosis. The predominant use of stapled sutures in the index surgery was consistent across all three grades of stenosis. Anastomosis closure with a circular stapling device has been linked to an increase in the incidence of stricture to as high as $30 \%$ when compared with hand-sewn anastomosis [14-16]. This risk, however, is independent of the stapler diameter.

Benign stenosis of colonic, rectal or colorectal anastomosis can occur from as early as within the first postoperative month to as late as several years after the surgery [19]. Most subjects were diagnosed with a stricture in the first postoperative year [4,7-13]. One case reported by Dieuruf et al had a stricture diagnosed 12 years after the surgery [6].

\section{Prior interventions}

Only four studies reported the use of alternative techniques to treat the stricture before considering them for EECI therapy. The case reported by Wallstabe et al underwent six balloon dilations over 10 weeks after identification of the stricture [4]. Four months after the first dilation, a second series of treatment with balloon dilation, triamcinolone injections, as well as transanal advancement flap anoplasty, was carried out without success. Of the seven cases in the retrospective case 
Endoscopic incision therapy for benign colorectal stricture 3

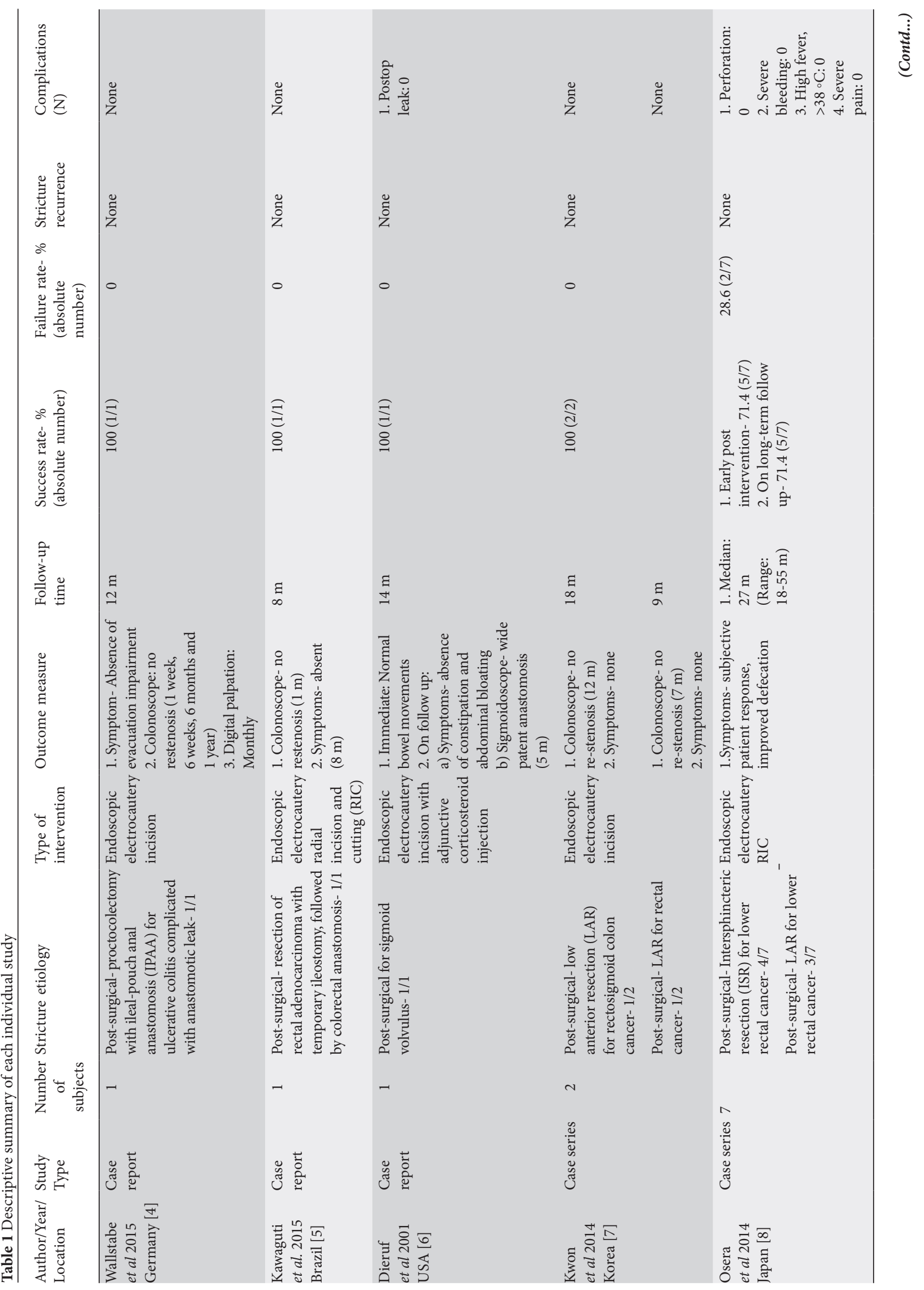




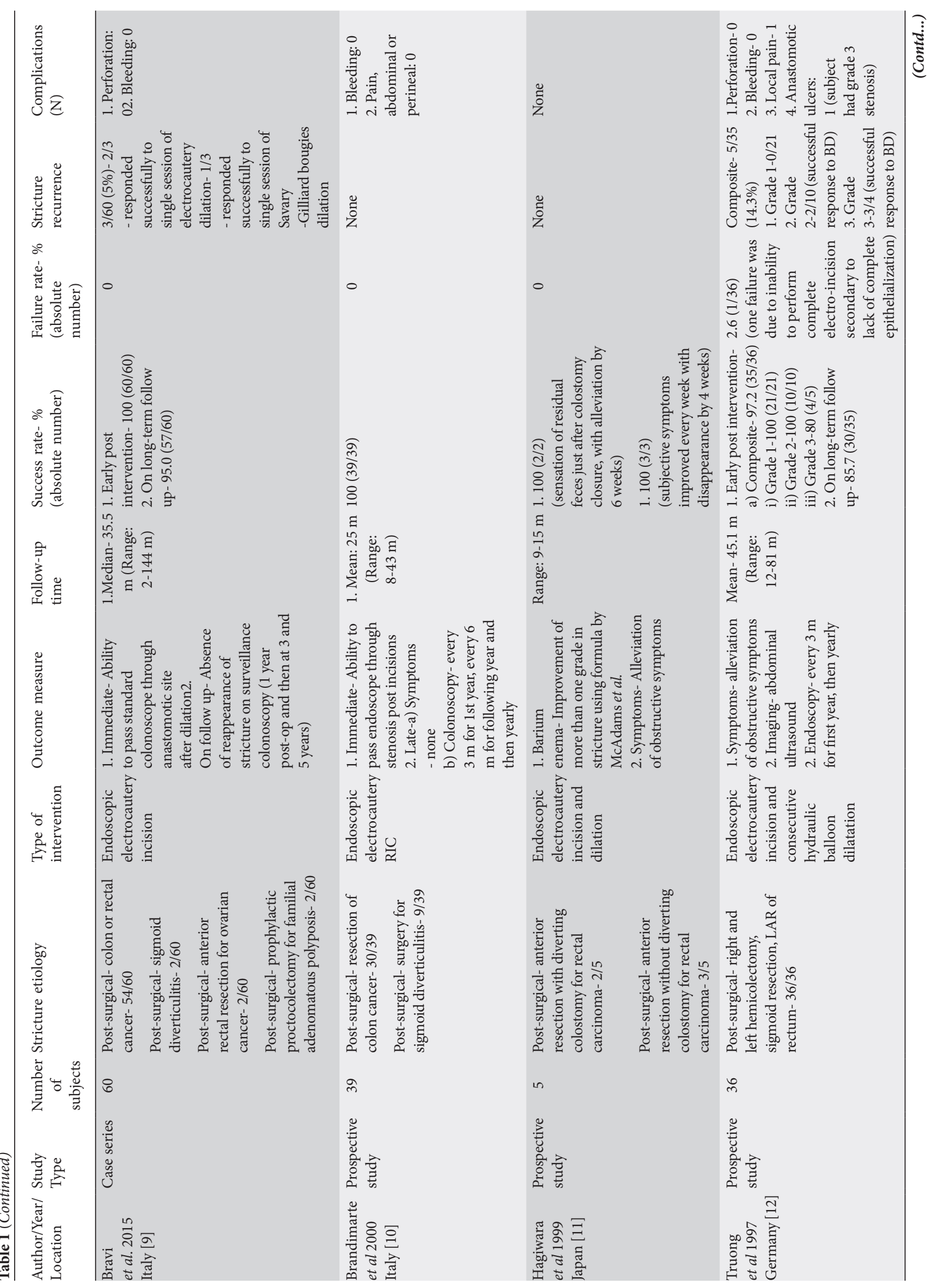




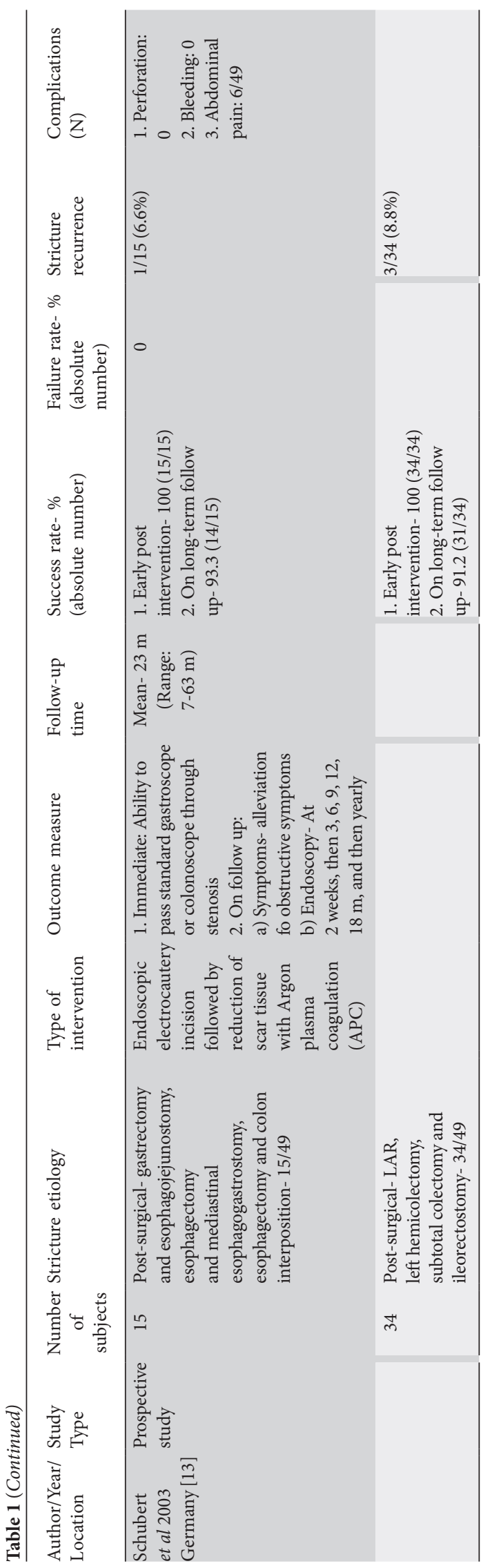

series by Osera $e t a l$, five underwent bougie dilation and the rest underwent balloon dilation at least more than twice before they were termed as refractory [8]. In the case reported by Dieruf $e t$ al, balloon dilation was performed to a maximum diameter of $18 \mathrm{~mm}$, but was followed by recurrence of abdominal distention after $48 \mathrm{~h}$ [6]. Hagiwara et al attempted two to three sessions of balloon dilations on all 41 patients, $12 \%(5 / 41)$ of whom failed to improve [11]. Balloon dilation of stenotic segments of the gastrointestinal tract has proved to be a convenient and less invasive treatment method. However, the majority of the patients require multiple sessions for the resolution of symptoms and recurrence is observed in about $18 \%$ of patients $[3,22]$.

All details regarding stricture etiology, location, criteria, characteristics, time interval to develop and interventions performed prior to consideration for EECI therapy have been summarized in Table 2.

\section{Procedure details}

\section{Anesthesia and bowel preparation}

Only four studies reported the type of anesthesia used for the procedure. Osera et al reported using conscious sedation via $2-3 \mathrm{mg}$ of IV midazolam and analgesia via $35 \mathrm{mg}$ of IV pethidine [8]. Bravi et al also used 5-10 mg of IV diazepam or 2.5-5 mg of IV midazolam for conscious sedation in 53 of the 60 patients included [9]. The remainder (7/60) opted out of receiving any conscious sedation for the procedure. No patient discomfort or pain was reported by either group of patients [9]. Two prospective studies by Truong et al and Schubert et al also utilized conscious sedation and/or analgesia $[12,13]$. The former used 5-10 mg of IV diazepam with $40 \mathrm{mg}$ of IV butyl-scopolamine and the latter used 4-10 $\mathrm{mg}$ of IV midazolam $[12,13]$. No complications as a result of conscious sedation were reported in the studies detailed above.

With regard to bowel preparation, investigators used a regimen of liquid diet, oral laxatives and cleansing enemas to achieve a quality of bowel preparation that would allow adequate visualization for the therapy $[9,10,12,13]$.

\section{Technical details}

All studies included in the review utilized the EECI technique, either alone or in combination with another modality. Three studies described the isolated use of EECI technique for stricture $[4,7,9]$. Wallstabe et al incised the stricture at 3 points using an ERCP sphincterotome and a precut knife [4]. Case reports by Kwon et al described incisions based on the stricture length and caliber determined either endoscopically or radiologically [7]. The length and depth of the incision were gauged by the endoscopist and never crossed the muscular layer. The electrocautery therapy lasted for about 5-10 min. An insulated tip (IT) knife and a bimodal electrocautery were used along with a $12 \mathrm{~mm}$ colonoscope [7]. A similar approach was used in the case series by Bravi et al, 


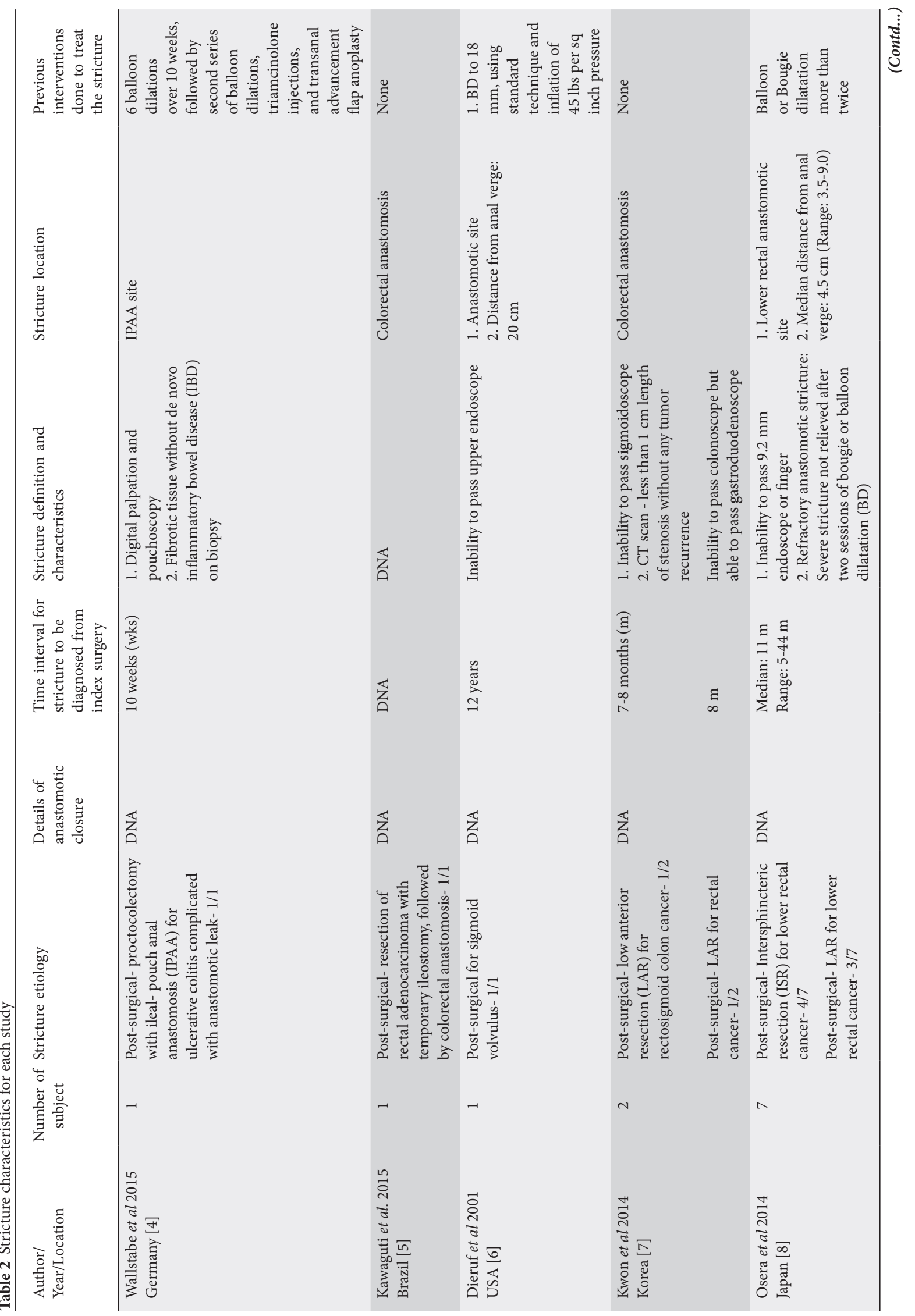




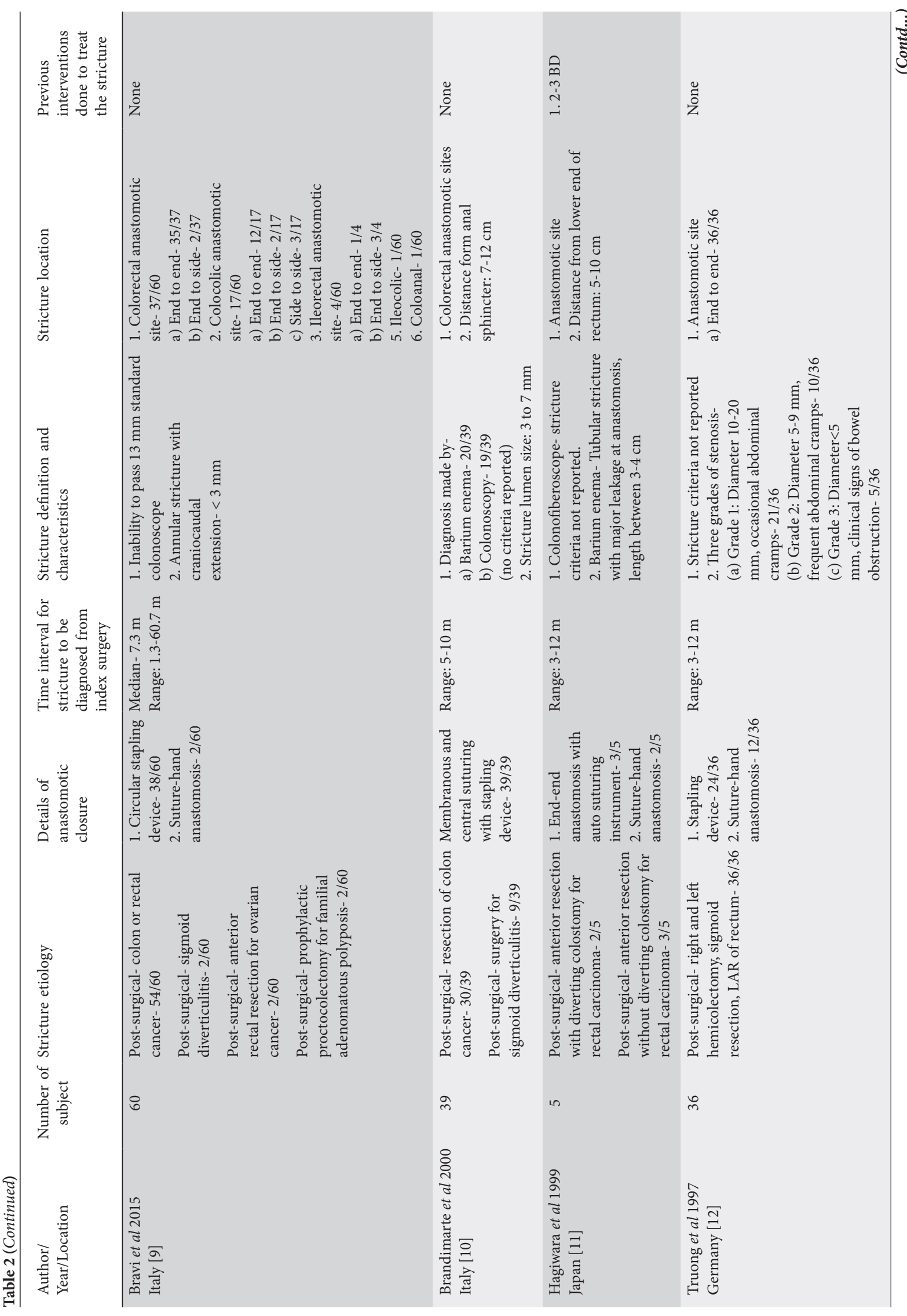




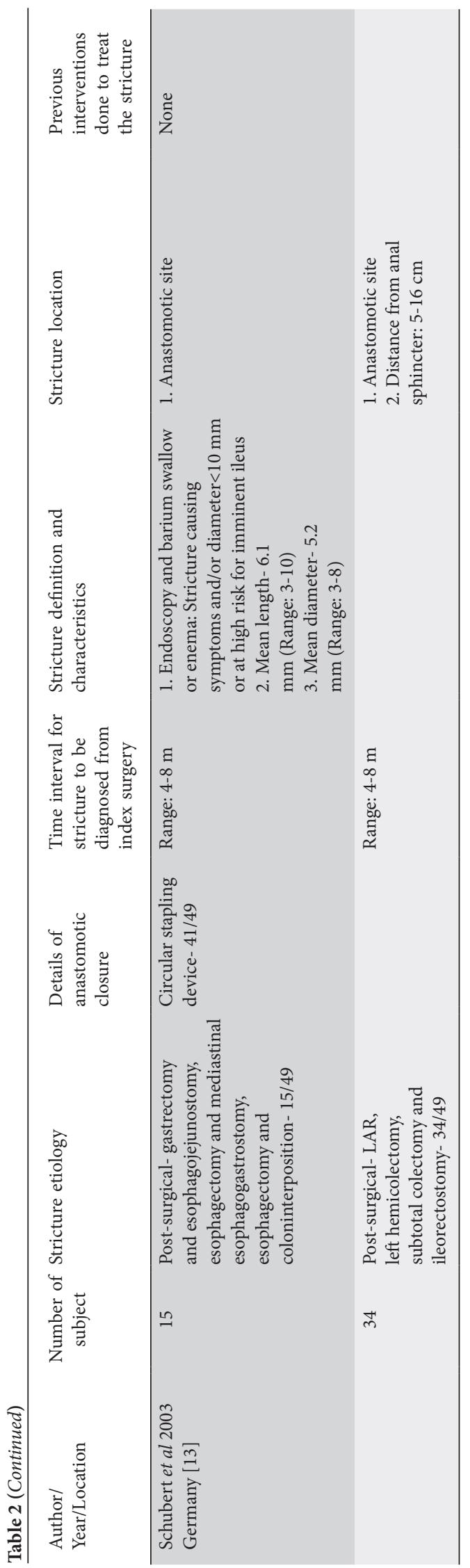

where two expert endoscopists performed the technique, making at least four incisions with a precut sphincterotome [9].

Three studies described using an endoscopic electrocautery RIC technique for stricture management. This is essentially same as EECI, but also involves resection of mucosal tissue in between the incisions $[5,8,10]$. Kawaguti et al and Osera et al both described making radial incisions with excision of the tissue in between the incisions along the lumen with an IT knife. The former took $12 \mathrm{~min}$ for the case and the latter reported a median duration of $18 \mathrm{~min}$ for the procedure [5,8]. In the retrospective case series by Osera et al, the majority of patients underwent a single inpatient procedure, with the median length of hospital stay being 3 days. The prospective study by Brandimarte et al utilized sphincterotome and bimodal electrocautery to make six radial incisions, the length and depth of which were operator dependent [10].

EECI with adjunctive corticosteroid injection was performed by Dieruf et al [6]. A 5-Fr needle knife was used to make incisions in four quadrants, with each cut being 5-8 mm in depth, resulting in a $20 \mathrm{~mm}$ luminal diameter. This was followed by injecting 7-10 mg of triamcinolone at each incision site using a sclerotherapy needle, up to a total of $35 \mathrm{mg}$.

EECI in combination with balloon dilation was described in two prospective studies [11,12]. Hagiwara et al used an endoscopic diathermic knife and a balloon dilator [11]. Two to three shallow radial incisions were made using repeated, short bursts in the stricture scar. A flexible tipped guide wire was then inserted, over which a balloon dilator was positioned at the stricture under fluoroscopy. Water-soluble contrast medium was used to inflate the balloon, which was then pulled out to have the stricture aligned in the middle. The balloon was left for 15-20 min and resulted in loosening of the scar. Of the five patients in this study, two who had a diverting colostomy underwent two sessions a week for two weeks and the remaining three patients without a colostomy had one treatment session only. In another prospective study by Troung et al, a similar technique of hydraulic balloon dilatation was performed after radial incisions were made [12]. The type of incision was dependent on whether the stenosis was central or eccentric. A higher number of consecutive dilatations were needed for a higher grade of stenosis.

Subjects enrolled in the prospective study by Schubert et al underwent incisions by a polypectomy snare, followed by reduction of the scar tissue in between the incisions by APC [13]. No more than one therapeutic session was needed for all subjects.

Details of the instruments used and techniques performed in each study have been summarized in Table 3.

\section{Outcome}

Outcomes were broadly classified based on the alleviation of symptoms and/or endoscopic evidence of absence of restenosis. Immediate evidence of a successful technique was described as ability to pass the endoscope through the stenosis site $[9,10,13]$. In subjects experiencing symptoms of obstruction as a result of 


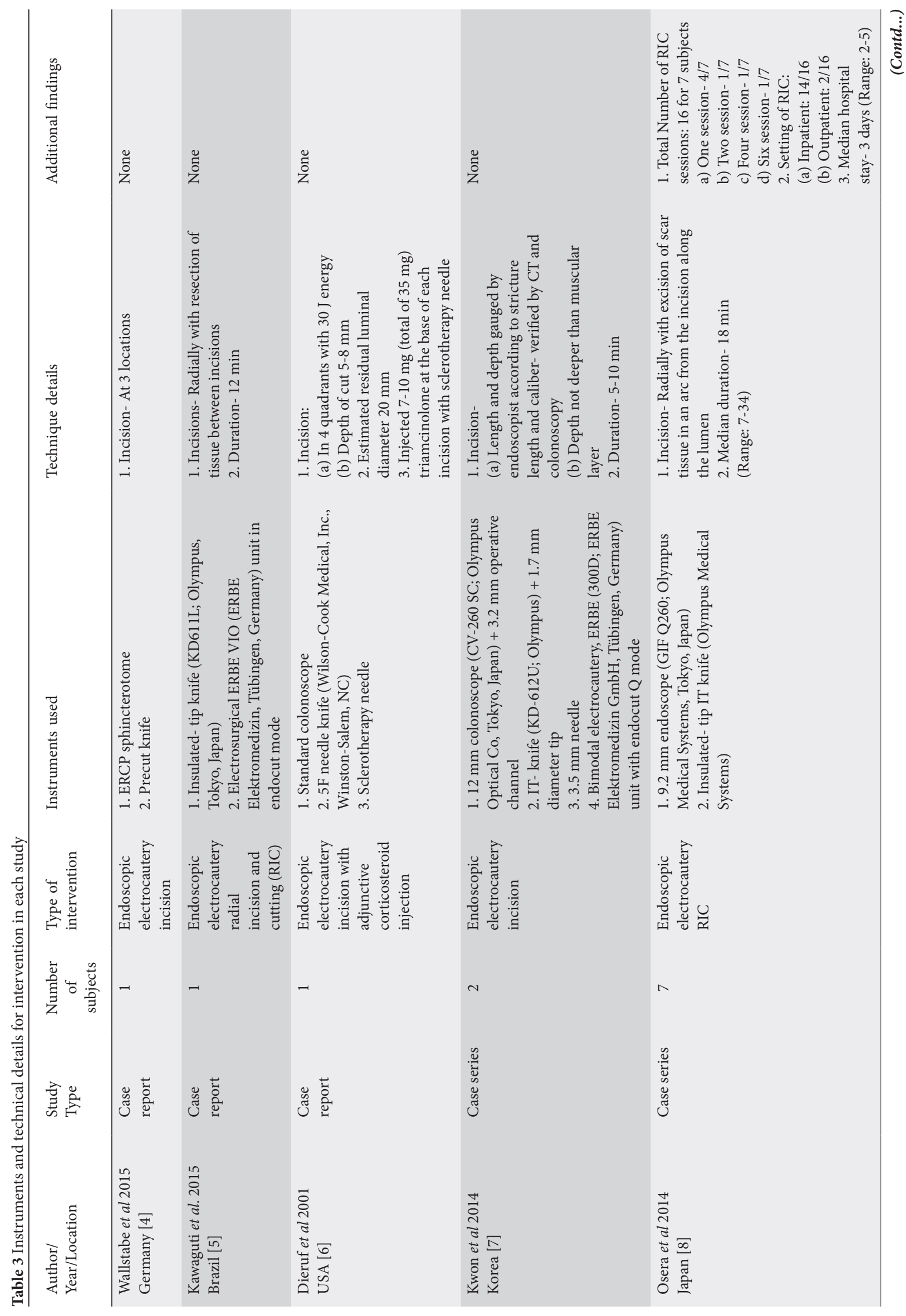




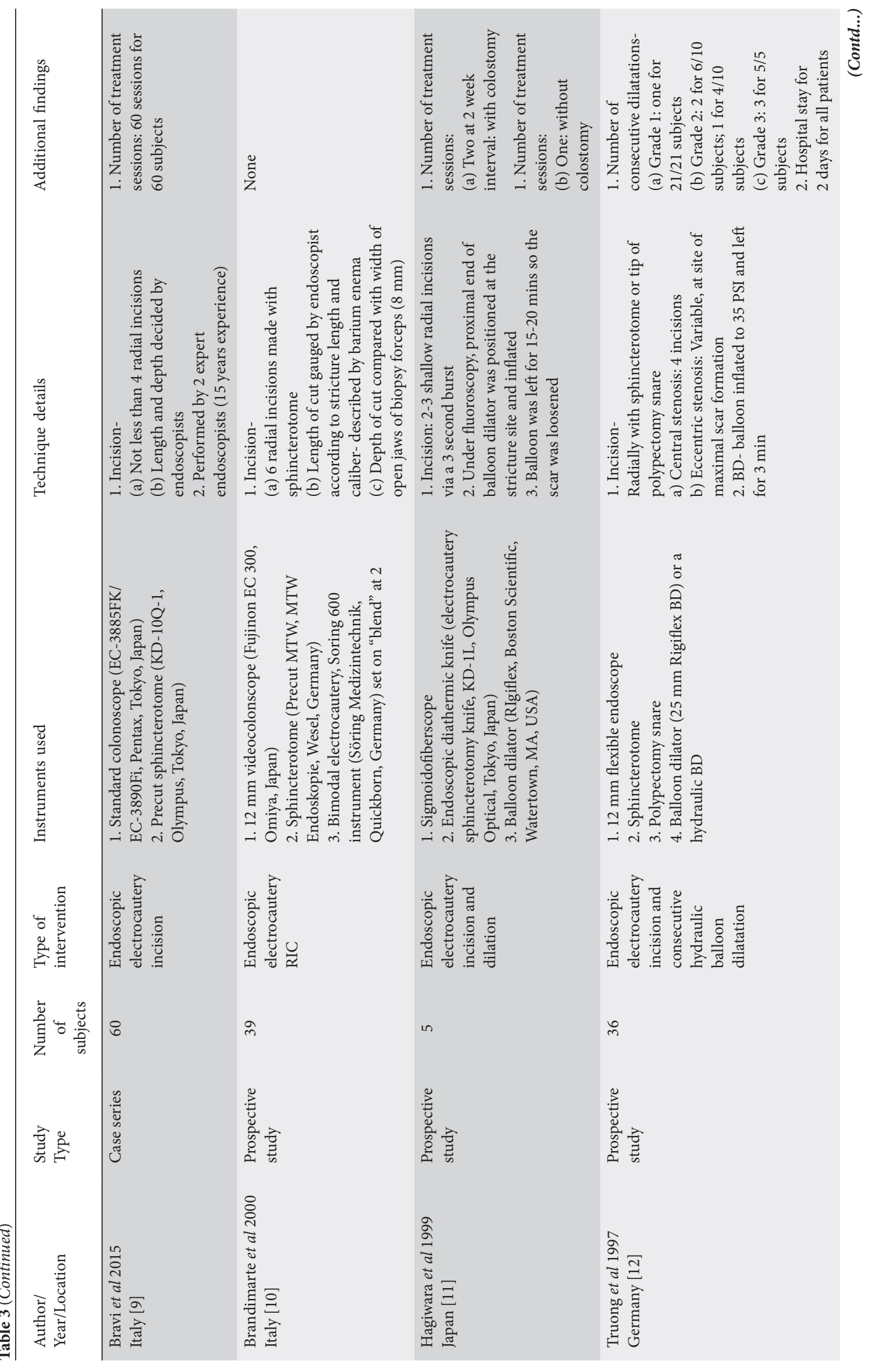




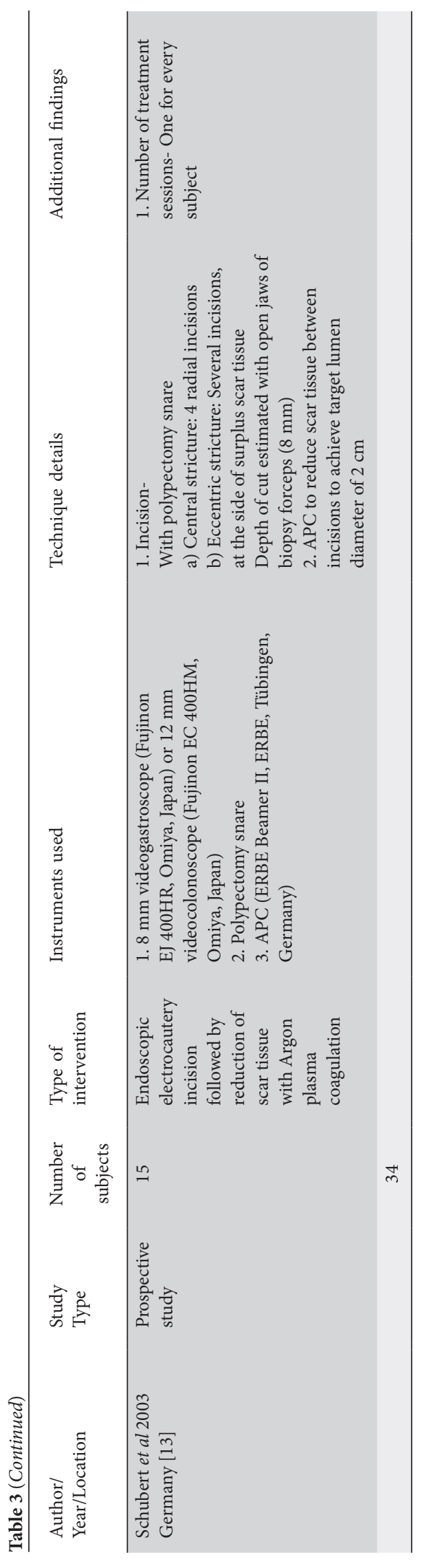

the anastomotic strictures, relief of symptoms like constipation, abdominal distension or abdominal pain was also considered a positive outcome measure for success of the technique. Serial follow ups were performed in all studies included in the review; they included evaluation of symptoms, digital palpation exams, endoscopic visualization and/or barium enema. The duration of follow up was variable, from a minimum of 8 months to a maximum of 12 years [7,9].

\section{Success}

All ten studies together had 186 subjects with lower gastrointestinal tract benign anastomotic stricture who underwent EECI, either alone or in combination with another treatment modality. Individually, 63 subjects underwent EECI alone $[4,7,9] ; 47$ subjects underwent endoscopic electrocautery with RIC $[5,8,10] ; 41$ subjects underwent combination therapy with EECI and balloon dilation [11,12]; 34 subjects had combination therapy with EECI and steroid injection [6]; and 1 subject had combination treatment with EECI and APC [13]. The overall success rate for early post procedure and on longterm follow up was $98.4 \%(183 / 186)$ and $93.0 \%(173 / 186)$, respectively. On long-term follow up, the success rate for EECI alone was 95.2\% (60/63), for EECI with RIC it was 95.8\% (45/47) and for combination treatment with EECI and balloon dilation it was $87.8 \%$ (36/41). Individual studies of combination treatment by EECI and steroid injection or APC reported success rates of $91.2 \%$ and $100 \%$, respectively $[6,13]$. Six studies reported a $100 \%$ success rate on long-term study-specific follow up [4-7,10,11].

Of 186 total subjects, 14 had a refractory stricture and 172 were treatment-naïve. Among the subjects with a refractory stricture, the success rate both early post-procedure and over long-term follow up was $85.7 \%(12 / 14)$. Among treatment naïve subjects, the early post-procedure and long-term success rates were $99.4 \%(171 / 172)$ and $93.6 \%(161 / 172)$ respectively Figure 1 and 2 depicts EECI of a benign colon stricture.

\section{Failure}

EECI, alone or in combination with another treatment modality, failed to achieve stricture dilation in $1.6 \%(3 / 186)$ of the total population [4-13]. All studies except two reported a failure rate of $0 \%$ in achieving post-intervention stricture dilation [4-13]. Osera et al reported that 2 of 7 subjects (28.6\%) failed to achieve stricture dilation, despite 4 and 6 sessions of endoscopic electrocautery with RIC, respectively [8]. In another study, the operator was unable to perform EECI and balloon dilation in 1 of 36 subjects $(2.8 \%)$, because of a lack of epithelialization of the stricture that increased the risk of perforation [12].

\section{Stricture recurrence}

Restenosis or recurrence of anastomotic stricture was identified as a result of development of symptoms and/or 


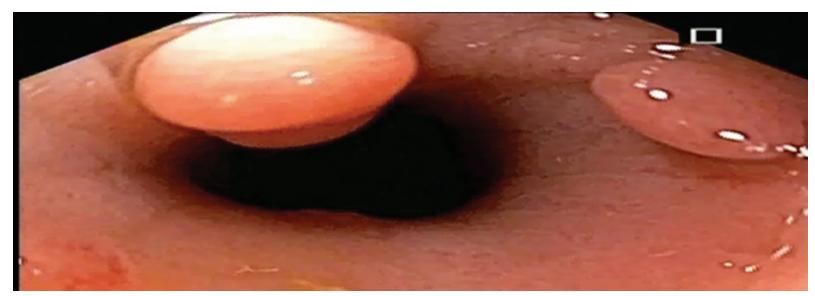

Figure 1 Colon stricture ( $3 \mathrm{~cm}$ long, benign, non inflammatory)

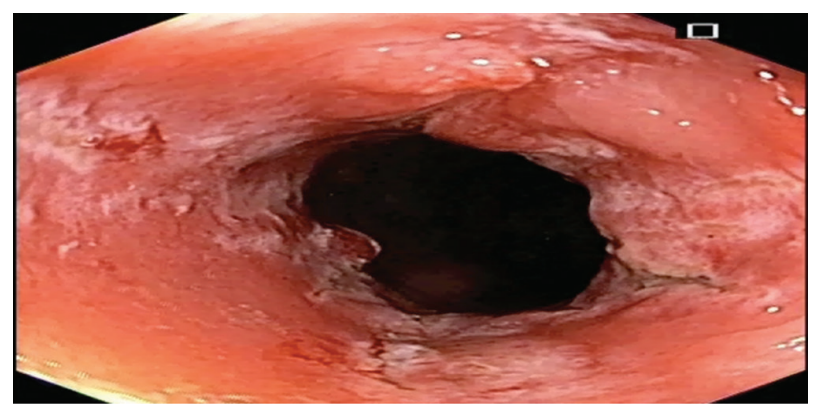

Figure 2 Post-endoscopic electrocautery incision of colon stricture

identification during follow-up endoscopic procedures. Bravi et al reported a 5\% (3/60) recurrence rate [9]. This was noted in three asymptomatic subjects; two were successfully treated with a single electrocautery dilatation and one was treated with a single session of Savary-Gilliard bougie dilation. Notably, in one of the subjects the primary anastomotic closure was performed using a circular stapling device. Truong et al had a stricture recurrence rate of $14.3 \%$ (5/35) [12]. Two patients who initially had a grade II stenosis and one patient with a grade III primary stenosis had a recurrent grade I restenosis; all were treated with a single balloon dilatation. Two patients with primary grade III stenosis were found to have grade II restenosis, which required two consecutive balloon dilation procedures with no further recurrences on follow up. Schubert et al had a recurrence rate of $8.8 \%$ (3/34) for lower gastrointestinal tract strictures [13]. All subjects required a mean number of 3.5 endoscopic sessions for treatment.

The stricture recurrence rate for subjects undergoing EECI alone was $4.8 \%$ (3/63), for endoscopic electrocautery with RIC it was $0 \%(0 / 45)$ and for combination therapy with EECI and balloon dilatation it was $12.5 \%$ (5/40). In our opinion, balloon dilatation in combination with EECI is likely to cause more edema, which disrupts the healing of the incision site and leads to a higher recurrence rate. Overall, the stricture recurrence rate for the cohort was $6.0 \%(11 / 183)$. The recurrence rate demonstrated in our cohort was much lower than that reported for balloon dilation alone (18\%) [3].

\section{Complications}

In comparison with standard treatment procedures for anastomotic strictures, such as balloon dilation, endoscopic incision techniques have been shown to have lower rates of perforation. In our review, eight studies reported a complication rate of $0 \%$ [4-11]. Of a total 186 subjects, only 7 (3.8\%) reported abdominal pain post-procedure. No other complication (bleeding, infection or perforation) was reported [4-13]. Schubert et al described 6 subjects (12.3\%) reporting some transitory abdominal pain [13]. Subjects were monitored for one day and discharged without any events, requiring no other intervention. Local abdominal pain was also described in 1 patient $(2.8 \%)$ by Truong et al [12]. It was attributed to the finding of a post-dilatation anastomotic ulcer, which healed with conservative management.

\section{Concluding remarks}

The incidence of benign colorectal anastomotic strictures is on the rise. The current standard for managing such strictures is primarily endoscopic balloon dilation therapy. The major pitfall of this modality is its high stricture recurrence rate and risk of perforation. The EECI technique, either alone or in combination with alternative treatment modalities such as steroid injection, balloon dilation or APC, is a promising new tool to treat both treatment-naive and refractory strictures. In our review, the overall success rate for EECIbased interventions for benign lower gastrointestinal tract strictures was $98.4 \%$, with a stricture recurrence rate of $6.0 \%$ and a composite complication rate of $3.8 \%$. The high success rate, lower stricture recurrence rate and lower incidence of complications in comparison to endoscopic balloon dilatation therapy make EECI the preferred option for short noninflammatory strictures. Head-to-head randomized controlled trials comparing EECI to endoscopic balloon dilation therapy are awaited before it can be considered as the standard therapy for the management of benign colorectal strictures.

\section{References}

1. Luchtefeld MA, Milsom JW, Senagore A, Surrell JA, Mazier WP. Colorectal anastomotic stenosis. Results of a survey of the ASCRS membership. Dis Colon Rectum 1989;32:733-736.

2. Polese L, Vecchiato M, Frigo AC, et al. Risk factors for colorectal anastomotic stenosis and their impact on quality of life: what are the lessons to learn? Colorectal Dis 2012;14:e124-e128.

3. Araujo SE, Furtado Costa A. Efficacy and safety of endoscopic balloon dilation of benign anastomotic strictures after oncologic anterior rectal resection. Surg Laparosc Endosc Percutan Tech 2008; 18:565-568.

4. Wallstabe I, Teich N. Successful endoscopic incision of pouchanal stricture in a patient with ulcerative colitis. Tech Coloproctol 2015; 19:429-430.

5. Kawaguti FS, da Costa Martins B, Nahas CS, et al. Endoscopic radial incision and cutting procedure for a colorectal anastomotic stricture. Gastrointest Endosc 2015;82:408-409.

6. Dieruf LM, Prakash C. Endoscopic incision of a postoperative colonic stricture. Gastrointest Endosc 2001;53:522-524.

7. Kwon JH, Han KH, Kim MH, et al. Two cases of electrocautery incision therapy using an insulated-tip knife for treatment of symptomatic benign short-segment colonic stenosis following 
colonic resection. Korean J Gastroenterol 2014;64:164-167.

8. Osera $\mathrm{S}$, Ikematsu $\mathrm{H}$, Odagaki $\mathrm{T}$, et al. Efficacy and safety of endoscopic radial incision and cutting for benign severe anastomotic stricture after surgery for lower rectal cancer (with video). Gastrointest Endosc 2015;81:770-773.

9. Bravi I, Ravizza D, Fiori G, et al. Endoscopic electrocautery dilation of benign anastomotic colonic strictures: a single-center experience. Surg Endosc 2016;30:229-232.

10. Brandimarte G, Tursi A, Gasbarrini G. Endoscopic treatment of benign anastomotic colorectal stenosis with electrocautery. Endoscopy 2000;32:461-463.

11. Hagiwara A, Sakakura C, Shirasu M, Torii T, Hirata Y, Yamagishi H. Sigmoidofiberscopic incision plus balloon dilatation for anastomotic cicatricial stricture after anterior resection of the rectum. World J Surg 1999;23:717-720.

12. Truong S, Willis S, Schumpelick V. Endoscopic therapy of benign anastomotic strictures of the colorectum by electroincision and balloon dilatation. Endoscopy 1997;29:845-849.

13. Schubert D, Kuhn R, Lippert H, Pross M. Endoscopic treatment of benign gastrointestinal anastomotic strictures using argon plasma coagulation in combination with diathermy. Surg Endosc 2003;17:1579-1582.

14. Beitler AL, Urschel JD. Comparison of stapled and hand-sewn esophagogastric anastomoses. Am J Surg 1998;175:337-340.
15. Petrin G, Ruol A, Battaglia G, et al. Anastomotic stenoses occurring after circular stapling in esophageal cancer surgery. Surg Endosc 2000;14:670-674.

16. MacRae HM, McLeod RS. Handsewn vs. stapled anastomoses in colon and rectal surgery: a meta-analysis. Dis Colon Rectum 1998;41:180-189.

17. Graffner H, Andersson L, Löwenhielm P, Walther B. The healing process of anastomoses of the colon. A comparative study using single, double-layer or stapled anastomosis. Dis Colon Rectum 1984;27:767-771.

18. Irvin TT, Goligher JC. Aetiology of disruption of intestinal anastomoses. Br J Surg 1973;60:461-464.

19. Pietropaolo V, Masoni L, Ferrara M, Montori A. Endoscopic dilation of colonic postoperative strictures. Surg Endosc 1990;4:26-30.

20. Choy PY, Bissett JP, Parry BR, Merrie AE. Stapled versus handsewn methods for ileocolic anastomosis. Cochrane Database Syst Rev 2007;(3):CD004320.

21. Simillis C, Purkayastha S, Yamamoto T, Strong SA, Darzi AW, Tekkis PP. A meta-analysis comparing conventional end-to-end anastomosis vs. other anastomotic configurations after resection in Crohn's disease. Dis Colon Rectum 2007;50:1674-1687.

22. Belverdere B, Frattaroli S, Crabone A, Viceconte G. Anastomotic strictures in colorectal surgery: treatment with endoscopic balloon dilation. G Chir 201;33:243-245.

Author Query

AQ1: Kindly cite figure 1 and 2 in text part 